\title{
The sand grain and the butterfly Instability in geodesy and geophysics
}

\author{
Helmut Moritz \\ Physical Geodesy, University of Technology, Graz, Austria
}

\begin{abstract}
The problems of convergence of series in celestial mechanics and of certain series in geodesy (Molodensky's series and spherical harmonics) show similar features, involving a curious instability. This is imaginatively expressed as the «butterfly effect» in chaos theory and the «sand-grain effect» for spherical harmonics. Similarly, the geodetic boundary-value problem (M.S. Molodensky, L. Hörmander) and the KAM problem in nonlinear dynamics have a common mathematical structure: a «hard» inverse function problem. Such interrelations are reviewed in the present paper.
\end{abstract}

Key words geodesy - geophysics - gravity field instability - chaos theory

\section{Introduction}

«We collectively wish to apologize for having misled the general educated public by spreading ideas about the determinism of systems satisfying Newton's laws of motion that, after 1960, were proved incorrect». This is what Sir James Lighthill, then President of the International Union of Theoretical and Applied Mechanics, said (Lighthill, 1994).

What Sir James meant is that the old belief that classical mechanics always leads to stable and predictable systems has been proved wrong by the modern general theory of nonlinear dynamical systems, now very popular by the name of Deterministic Chaos (e.g., Schuster 1988) or simply Chaos Theory. From the enormous «chaos» of books on this subject my favorites are Hilborn (1994) and Jackson

Mailing address: Prof. Helmut Moritz, Physical Geodesy, University of Technology, Steyrergasse 30, A-8010 Graz, Austria; e-mail: moritz@fpgeods02.tu-graz.ac.at
(1991, with many references). Chaos theory is particularly popular with computer fans since it generates pictures of wonderful exotic beauty; $c f$. the programs and diskettes in the books (Herrmann, 1994; Korsch and Jodl, 1994).

Modern chaos theory is said to start with the work of A.N. Kolmogorov and V.I. Arnold (cf. Arnold and Avez, 1968) and by Lorenz (1963). Its principles and implications were, however, already fully understood, by the great mathematician Henri Poincaré; cf. Poincaré (1908, reprint, p. 68), also quoted in Moritz (1995). A special case, the «problem of small denominators», had already been known for a long time in celestial mechanics, but it was only the mathematician Heinrich Bruns (1884) who shortly before Poincare described the formidably mathematical implication of this special case; $c f$. also Wintner (1941).

\section{Convergence of astronomical series}

Let us briefly outline the work of Bruns in a simplified manner, following Moritz (1969).

Problems of convergence already arise in the first approximation, in the well-known case 
of linear perturbations. Planetary perturbation equations are of the type

$$
\frac{d x}{d t}=\sum_{m=0}^{\infty} \sum_{n=0}^{\infty} \mu^{m+n} \cos (m \lambda-n) t,
$$

where $x$ is a typical orbital element which slowly changes because of the effect of attraction of a perturbing planet; $t$ is the time, and $\lambda$ and $\mu$ are given positive constants with $\mu<1$, so that the series (2.1) will be uniformly convergent.

The example (2.1) is very instructive because it exhibits in a simple manner several interesting and even strange features that arise, in some way or other, in many convergence problems. Similar cases are considered by Brouwer and Clemence (1961) for planetary perturbations and by Moritz and Mueller (1987) for precession and nutation.

Equation (2.1) can be directly integrated to give, for the initial condition $x(0)=0$,

$$
x=B t+\sum_{m} \sum_{n} \frac{\mu^{m+n}}{m \lambda-n} \sin (m \lambda-n) t,
$$

which we shall call «Bruns' series». The sine terms arise from integrating the corresponding cosine terms in (2.1). All terms for which

$$
m \lambda-n=0
$$

must be excluded from the sum in (2.2). For such terms we have

$$
\cos (m \lambda-n) t=1
$$

the integral of which is $t$, so that the term $B t$ represents the integral of the sum of all these constant terms.

The value of the constant $B$ depends on whether $\lambda$ is a rational or an irrational number. If $\lambda$ is irrational, then the condition (2.3) is satisfied only for $m=n=0$. The corresponding term in (2.1) is 1 , so that we have

$$
B=1 \text { for } \lambda \text { irrational. }
$$

If $\lambda$ is rational, however, then $\lambda$ has the form

$$
\lambda=\frac{p}{q},
$$

where $p$ and $q$ are relatively prime integers, so that the condition (2.3) is satisfied for

$$
m=h q, \quad n=h p
$$

with $h=0,1,2,3 \ldots$ By (2.1) we then have infinitely many constant terms, so that now

$$
B=\sum_{h=0}^{\infty} \mu^{h(p+q)} \quad\left(\text { for } \lambda=\frac{p}{q} \text { rational }\right)
$$

Although for every $\lambda$ the solution may be written in the form (2.2), it shows some rather strange features: if $\lambda$ is a rational number, then the series (2.2) may be shown to be convergent, because then the small divisors $m \lambda-n$ will not become «too small». If $\lambda$ is irrational, then the series may be convergent or divergent in such a way that in every interval

$$
\lambda_{0}<\lambda<\lambda_{0}+\varepsilon
$$

of arbitrarily small length $\varepsilon$, there always exist infinitely many values $\lambda$ for which the series (2.2) converges and infinitely many values $\lambda$ for which this series diverges. This is the essential result of Bruns (1884).

Thus the convergence or divergence of the series (2.2) depends on the value $\lambda$ and is quite different for two values of $\lambda$ that are arbitrarily close to each other. We thus have Bruns' Theorem: If Bruns' series (2.2) converges for a certain value $\lambda_{0}$, it is always possible to find an arbitrary small value $\varepsilon$ so that for $\lambda=\lambda_{0}+\varepsilon$ the series diverges, and vice versa.

Thus, since $\lambda$ is known empirically only to a certain accuracy (within $\lambda-\varepsilon$ and $\lambda+\varepsilon$, say), the question whether Bruns' series is convergent or not, is practically meaningless!

Remark. H. Bruns is also the author of «The figure of the Earth» (1878) and the discoverer of «Bruns' formula» for the geoid, well-known in geodesy; $c f$. Moritz (1980, p. 14). 


\section{Asymptotic series}

In perturbation theory applied in many disciplines of mathematics, mathematical physics, astronomy, etc., the solution is tentatively expressed as a formal power series with respect to a «small parameter» $k$ :

$$
x=x_{0}+x_{1} k+x_{2} k^{2}+x_{3} k^{3}+\ldots,
$$

where $x$ stands for the quantities that we wish to determine, and the $x_{i}$ are functions that depend on the data.

Expansions of the type (3.1), whether they are convergent or divergent, are always asymptotic series; that is, we have

$$
\lim _{k \rightarrow 0} \frac{1}{k^{n}}\left[x-\left(x_{0}+x_{1} k+x_{2} k^{2}+\ldots+x_{n} k^{n}\right)\right]=0
$$

for every integer $n$. This follows from a property of asymptotic series: a formal power series (3.1) with arbitrary finite coefficients $x$, is asymptotic (Erdélyi, 1956).

Our astronomical series (2.2) have the form (3.1) for $k=1$.

Poincaré (1987, vol. 2, pp. 1ff, 452ff) has established that the astronomical series (of Bohlin and others) are asymptotic and in general divergent.

This does not mean that such divergent series cannot be applied in practice. Mathematicians use the well-known Stirling series to compute the gamma function; physicists solve nonlinear oscillation problems, astronomers calculate precise planetary orbits, and geodesists determine height anomalies and deflections of the vertical, all by means of asymptotic series that are, mathematically speaking, divergent. Such series can be used if the first terms decrease rapidly enough for their sum to provide a good approximation to the function to be calculated; it will not matter practically if the neglected higher terms start increasing again.

The practical use of divergent series needs, however, to be justified. An arbitrarily accurate approximation can be obtained only with convergent series; with divergent asymptotic series, this error cannot be reduced below a certain limit. It must be investigated whether this limit is small enough to be in keeping with the desired accuracy. The answer to such questions requires a considerable understanding of the convergence or divergence behavior of the series under consideration.

By such considerations of convergence or divergence, Poincaré (1890) established for the first time the instability and chaotic behaviour of many (in a certain sense, of most) problems of classical mechanics, and thus founded chaos theory.

Precise conditions for the stability or chaoticity of nonlinear dynamic systems have been given since about 1960 by the famous Russian mathematician A.N. Kolmogorov, his coworker V. I. Arnold and the German mathematician J. Moser (the «KAM-theorem»). They showed in a very general way that convergence or divergence, stability or instability of nonlinear dynamical systems depends on number-theoretical properties (similar to rationality or irrationality) of a certain parameter $\lambda$, thus extending the results of Bruns to nonlinear problems. Thus convergence or divergence may change by going from one value of the parameter $\lambda$, to an infinitely close value $\lambda+\varepsilon$.

Nevertheless, as mentioned above, asymptotic series may be used very well in numerical practice even if they are, strictly speaking, divergent. This is why they are sometimes called «semiconvergent».

Starting with my 1969 paper, I tried to apply these ideas to the well-known Molodensky series solution of the geodetic boundary-value problem. The paper (Moritz, 1971) shows that Molodensky's series is an asymptotic series, and (Moritz, 1973) investigates its convergence. Vastly superior to these rather dilettante efforts is the investigation of the famous Swedish mathematician Hörmander (1976), using the powerful tools of modern mathematics, but his results hold only for a surface that is much smoother than the Earth. Sansò $(1976,1977)$ obtained similar results with simpler mathematics, but only for a non-rotating Earth. All this work is reviewed in the book (Moritz, 1980).

Is it not remarkable that the basic mathematical structure, a «hard» inverse problem of modern functional analysis, is the same in the KAM theorem and in Hörmander's work? 


\section{The Lorenz attractor}

Another pioneer of modern chaos theory, Lorenz (1963) discovered a very particular form of instability in meteorology. Everyone knows that weather prediction is extremely unreliable beyond, say, three days. Already the great Poincaré (1908) had understood perfectly the underlying mathematical-physical situation, as remarked above.

Lorenz simplified the hydrodynamic-thermodynamics equations of meteorology to obtain a simple system of three differential equations for three unknown functions $x(t), y(t)$, $z(t)$ of time $t(x, y, z$ are not Cartesian coordinates but represent the system's state):

$$
\begin{aligned}
& \frac{d x}{d t}=-3 x+3 y, \\
& \frac{d y}{d t}=-x z+26.5 x-y, \\
& \frac{d z}{d t}=x y-z ;
\end{aligned}
$$

the nonlinearities $x y$ and $x z$ are essential. Solving the three equations by numerical integration, Lorenz found that a very small difference in the initial conditions will cause large deviations in the corresponding trajectories (fig. 1). Furthermore, the trajectories show a strange attractor of butterfly-like shape. A trajectory will spend some time orbiting around one «wing» of the butterfly and then unpredictably change to the other «wing», orbiting around it a «random» number of times before jumping back to the first wing, and the game continues indefinitely.

It is particularly impressive to look at a computer animation following the motion of a number of particles which are first undistinguishingly close to each other. In the course of time the particles separate, until they are randomly distributed over the computer screen.

This is a typical case of unstable motion: stability means that small causes produce small changes, whereas in instability, small causes may produce large effects.

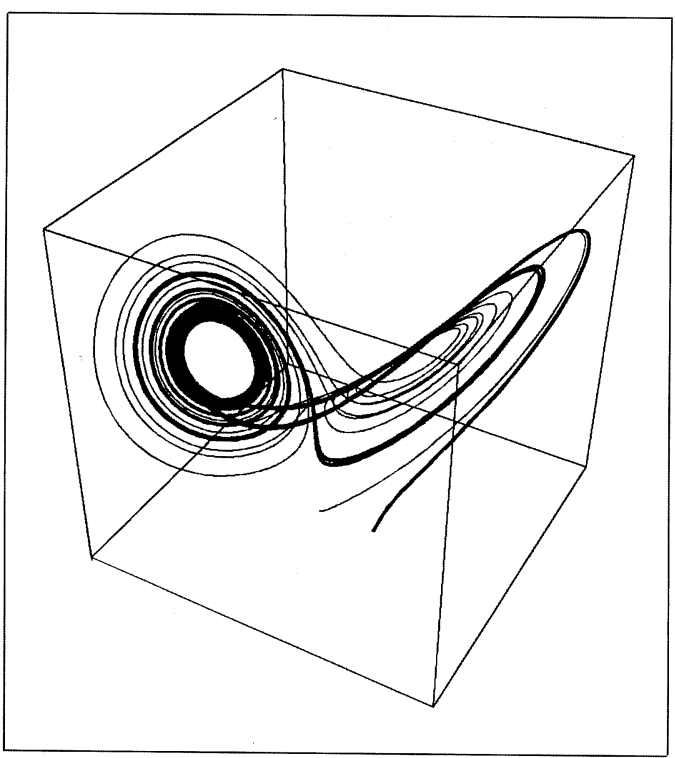

Fig. 1. The Lorenz attractor.

Lorenz has picturesquely described this phenomenon as the butterfly effect: the flight of a butterfly in Austria may (in principle) cause a tornado in America.

In the geodynamo theory of the core's electromagnetic field, a similar attractor might exist, and the change of our particle (representing the system's state) from one wing to the other may correspond to the unpredictable jump of polarity of the Earth's magnetic field every million years or so on the average.

As a matter of fact, there are also other applications of nonlinear system theory in geophysics, one of the most important being the application to earthquake theory by KeilisBorok and co-workers; $c f$. Keilis-Borok (1990).

\section{The convergence of the spherical har- monics expression of the geopotential}

The development of the external gravitational field into a series of spherical harmonics has long been known to be convergent outside and on the smallest sphere $\sigma_{1}$ around the origin 
O that just touches the Earth's surface $E$ (fig. 2). It would be convergent even at the Earth's surface if the analytical continuation of the external potential exists and is regular to the second limit sphere $\sigma_{2}$ which just touches the Earth's surface from the inside.

Assuming the latter, a pointlike grain of sand $S$, of arbitrarily small mass $m$, lying on the Earth's surface, will change convergence into divergence since it produces a potential

$$
v=\frac{G m}{l},
$$

where $l$ is the distance from $S$ and $G$ is the Newtonian gravitational constant. At $S$, the potential (5.1), and hence also the analytical continuation of the total external potential, will have a singularity. Hence this analytical continuation will no longer be regular down to $\sigma_{2}$, so that the external potential will no longer converge over the whole Earth's surface $E$. The mass $m$ might be as small as we like, e.g., $10^{-10} \mathrm{~kg}$.

This simple consideration of Moritz (1961) was supplemented by Krarup (1969) by showing that also the converse is true: not only can a sand grain change convergence into divergence, but an arbitrarily small change of the external potential may also change divergence

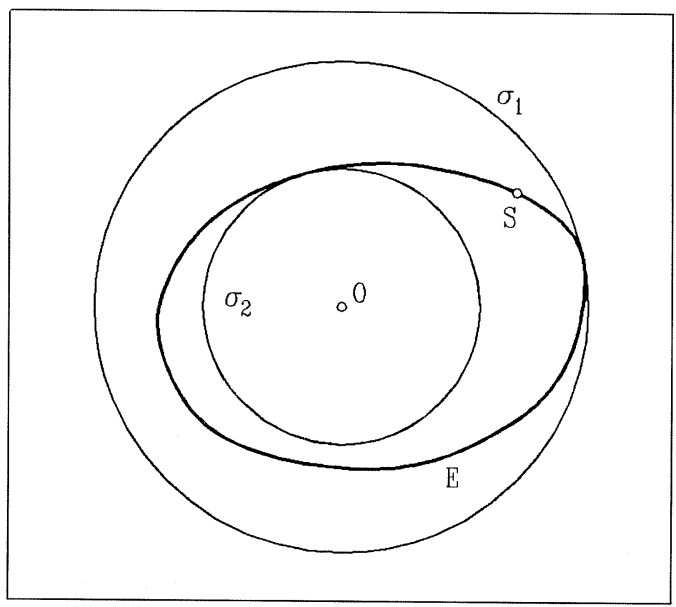

Fig. 2. The limit spheres $\sigma_{1}$ and $\sigma_{2}$. into convergence! This is the sand-grain effect in gravimetry; Krarup (1969) called it «Runge's Theorem» because Runge proved a similar theorem for analytic functions of a complex variable. For more details $c f$. Moritz (1978) and the book (Moritz, 1980, sections 6-8).

«Arbitrarily small» means arbitrarily small, e.g., changing gravity along the Earth's surface in such a way that the change nowhere exceeds $10^{-10}$ mgals. This is obviously beyond measuring accuracy, so that the question of convergence or divergence of the geopotential at the Earth's surface is empirically irrelevant. Thus, for practical purposes, the spherical harmonic series of the geopotential can always be considered to be convergent at the Earth's surface.

Thus, paraphrasing the statement of Sir James Lighthill quoted at the beginning, I should like to say: «We theoretical geodesists wish to apologize to the geodetic and geophysical community for the false impression given by us, that the question of mathematical convergence of the geopotential at the Earth's surface has any practical significance».

\section{REFERENCES}

Arnold, V.I. and A. Avez (1968): Ergodic Problems of Classical Mechanics (Benjamin, New York).

Brouwer, D. and G.M. Clemence (1961): Methods of Celestial Mechanics (Academic Press, New York), p. 297.

BRuns, H. (1884): Bemerkungen zur Theorie der allgemeinen Störungen, Astron. Nachr., 109, 216-222.

ERDÉlYI, A. (1956): Asymptotic Expansions (Dover, New York) p. 22.

Herrmann, D. (1994): Algorithmen für Chaos und Fraktale (Addison-Wesley, Bonn).

Hilborn, R.C. (1994): Chaos and Nonlinear Dynamics (Oxford University Press).

HÖRMANDER, L. (1976): The boundary problems of physical geodesy, Arch. Rat. Mech. Anal., 62, 1-52.

JACKson, E.A. (1991): Perspectives of Nonlinear Dynamics, (Cambridge University Press), 2 vols.

KEILIS-BoROK, V.I. (Ed.) (1990): Intermediate-term earthquake prediction: models, algorithms, worldwide tests Phys. Earth Planet. Inter. (Elsevier, Amsterdam), 61 (special issue).

Korsch, H.J. AND H.J. JodL (1994): Chaos - A Program Collection for the PC (Springer, Berlin).

KRARUP, T. (1969): A contribution to the mathematical foundation of physical geodesy, Dan. Geod. Inst., Copenhagen, Publication No. 44. 
LightHILl, J. (1994): Chaos: a historical perspective, in Nonlinear Dynamics and Predictability of Geophysical Phenomena, Geophysical Monograph 83, IUGG, vol. 18, American Geophysical Union, Washington, D.C.

LORENZ, E.N. (1963): Deterministic nonperiodic flow, J. Atmos. Sci., 20, 130-141.

MoRITZ, H. (1961): Über die Konvergenz der Kugelfunktionsentwicklung für das Aussenraumpotential an der Erdoberfläche, Österr. Z. Vermess., 49, 11-15.

MoRITZ, H. (1969): Convergence problems in celestial mechanics and physical geodesy, in Proceedings, IV Symposium on Mathematical Geodesy, Trieste, 81-94.

MoRITZ, H. (1971): Series solutions of Molodensky's problem, Publ. Deut. Geod. Komm, A-70, München.

MoRITZ, H. (1973): On the convergence of Molodensky's series, Boll. Geod. Sci. Affini, 32, 125-144.

MoRITZ, H. (1978): On the convergence of the sphericalharmonic expansion for the geopotential at the Earth's surface, Boll. Geod. Sci. Affini, 37, 363-381.

Moritz, H. (1980): Advanced Physical Geodesy (Wichmann, Karlsruhe), 2nd edition, 1989.

MoRITz, H. (1995): Science, Mind and the Universe -
An Introduction to Natural Philosophy, (Wichmann, Heidelberg), p. 243.

MoritZ, H. and I.I. Mueller (1987): Earth Rotation Theory and Observation (Ungar, New York), p. 93.

PoINCARÉ, H. (1890): Sur le problème des trois corps et les équations de la dynamique, Acta Mathematica, 13, 1-270.

PoInCARÉ, H. (1908): Science et Méthode (Flammarion, Paris) reprint in English by Dover, New York.

PoINCARÉ, H. (1987): Les Méthodes Nouvelles de la Mécanique Céleste, 3 vols., Albert Blanchard, Paris (reprint of the original edition from 1892, 1893 and 1899).

SANSÒ, F. (1976): Discussion on the existence and uniqueness of the solution of Molodensky's problem in gravity space, Accad. Naz. Lincei, Rend. Sci. Fis. Mat. Nat., 61, 260-268.

SANSÒ, F. (1977): The geodetic boundary value problem in gravity space, Mem. Accad. Naz. Lincei, 14, 39-97.

SCHUSTER, H.G. (1988): Deterministic Chaos (VCH Weinheim, Germany), 2nd edition.

WinTNER, A. (1941): The Analytical Foundations of Celestial Mechanics (Princeton University Press), p. 407. 